\title{
El primer catálogo español de especies protegidas (1896): análisis de su contenido y autoría de Graells
}

\author{
J. J. Ferrero-García*
}

Las primeras disposiciones legales importantes en materia de conservación de las especies silvestres surgieron en la segunda mitad del siglo XIX, siempre en relación con la protección de las aves (MacPherson, 1909), como por ejemplo el denominado An Act for the Preservation of Sea Birds (Kettilby, 1869). En 1884, en el Primer Congreso Ornitológico Internacional, celebrado en Viena, se sugirió la conveniencia de un convenio internacional que salvaguardara las aves en general, y que se basara en la prohibición de su caza durante la época de cría (Oustalet, 1884). En parte, este proceso cristalizó al constatarse la necesidad de promover la protección de la fauna valiosa para el sector agrario, debido a su papel auxiliar en la lucha contra las plagas del campo, reavivándose el antiguo debate sobre la presunta utilidad o perjuicio de muchas especies (Torres-Vila et al., 2009). Así, estas acciones desembocaron en nuevas iniciativas supranacionales, entre ellas la firma por doce naciones europeas, en 1902, de la Convención Internacional para la Protección de las Aves Útiles a la Agricultura, vigente durante toda la primera mitad del siglo XX (SEO, 1956; van Heijnsbergen, 1997; Boardman, 2006).

Uno de los estados firmantes de la Convención de 1902 fue España, que también había elaborado su propia normativa proteccionista (Peña, 1905; Calleja, 1926), mediante la aprobación de la Ley de 19 de septiembre de 1896, de protección de los pájaros y, sobre todo, de la Real Orden de 25 de noviembre de 1896 (en adelante RO), por la que se creaba un catálogo de aves protegidas (Ministerio de Fomento, 1896). La RO estaba constituida por una lista de ciertos grupos de aves (Alaudidae,
Emberizidae, Fringillidae, Laniidae, etc.) cuya caza se prohibía durante la época de cría, y, por otro listado más detallado para cuyos componentes se establecía una protección absoluta. En definitiva, había nacido la primera norma que amparaba estrictamente a una porción de la fauna silvestre española, y cuyo contenido permaneció inalterado durante gran parte del siglo XX, al integrarse sin variación en las leyes que la sustituyeron pocos años después (Ferrero-García, 2010).

Con la RO España se había situado al mismo nivel que otros países preocupados por el estado de conservación de sus aves, pudiéndose asegurar que una parte de su avifauna estaba, al menos sobre el papel, bien protegida (MacPherson, 1909). Sin embargo, existen muy pocos estudios que traten sobre su contenido y circunstancias, en contraposición con la atención que, en otras naciones, se ha prestado al análisis de sus primeras normas de conservación de la vida silvestre (e.g., Barclay-Smith, 1959; Marchant $\&$ Watkins, 2009). Por ello, en este trabajo se quiere realizar un acercamiento a la RO, ofreciendo una breve sinopsis de los grupos de aves que amparaba durante todo su ciclo vital, y examinando su calidad técnica en el contexto de la ciencia decimonónica. Asimismo, se ha comprobado documentalmente que el autor fundamental de esta norma fue uno de los más conocidos naturalistas españoles del siglo XIX: Mariano de la Paz Graells (1809-1898).

Para interpretar correctamente la nomenclatura científica manejada en la RO se ha utilizado el Prontuario de la avifauna española (Bernis, 1954), donde se recogen las principales sinonimias empleadas en España en el periodo 1850-1950. Con el mismo fin, y para obtener además un resumen de los

\footnotetext{
${ }^{*}$ Consejería de Agricultura y Desarrollo Rural, Junta de Extremadura. C/ Arroyo de Valhondo, 2. 10071 Cáceres. España. E-mail: juanjo.ferrero.g@gmail.com.
} 
conocimientos existentes en aquella época sobre la ornitofauna hispana, se ha consultado Aves de España (Arévalo, 1887), que está considerado como el mejor compendio de la ornitología española hasta bien entrado el siglo XX (Bernis, 1998; Garrido, 2006). De hecho, era una de las pocas obras nacionales bien valoradas ya a finales del XIX por prestigiosos autores extranjeros (Irby, 1895). Igualmente, se ha examinado el Catálogo de las aves de España, Portugal é Islas Baleares (Reyes, 1886) que, junto con el anterior libro de Arévalo, constituyen las dos primeras recopilaciones en las que se intenta dar una visión general de la situación de la avifauna española (Tellería, 2004). Complementariamente, se han consultado algunos de los grandes clásicos sobre las aves de Europa publicados a lo largo del siglo XIX (e.g., Temminck, 1815), así como el Tratado de las aves insectívoras cuya caza está prohibida en España (Peña, 1905), una documentada obra de divulgación sobre las especies protegidas escrita hace más de una centuria. Asimismo, ha sido imprescindible la información aportada por el propio texto de la RO (Ministerio de Fomento, 1896) y, también, la encontrada en el Archivo central del Ministerio de Medio Ambiente, y de Medio Rural y Marino (en adelante AMRM), en el Área de Medio Rural y Marino.

En general, la RO se refería de manera individual (empleando la denominación común y la científica) a casi todas las especies, pero en el caso de las rapaces nocturnas únicamente utilizaba los nombres vernáculos en español. Por tanto, en este trabajo se han sumado solo las Strigiformes que, inequívocamente, se podrían en aquella época designar con dichos nombres comunes, y que además son las únicas aves de presa nocturnas que incluía Peña (1905) dentro de la RO. Asimismo, con respecto a los miembros de la familia Picidae, excepto para Jynx torquilla, la RO únicamente mencionaba la inclusión de los denominados géneros Gecinus, Dryocopus, Picus y Apternus, por lo que en este texto se han considerado todos los pájaros carpinteros que aparecían citados en España por Reyes (1886) o Arévalo (1887).

Con estas salvedades, es posible establecer que la RO amparaba estrictamente (teniendo en cuenta los nombres y sinonimias originales) a 82 especies de aves, número que se podría elevar a 84 al introducir los cambios taxonómicos realizados desde finales del siglo XIX hasta la actualidad. De esta manera, hay algunos taxones que en el texto original eran considerados como varias especies (es el caso de Motacilla alba, que aparecía dividida en M. alba y M. lugubris), pero más frecuentemente, determinados taxones incluidos originariamente en la RO han sido escindidos, en la actualidad, en más de una especie (verbigracia, Anthus spinoletta y Anthus petrosus eran denominados indistintamente en la RO como Anthus aquaticus). En cualquier caso, de las 84 especies actuales, 59 son Passeriformes (un 70\%) pertenecientes a más de una docena de familias (Hirundinidae, Sylviidae, Muscicapidae, Paridae, etc.). El resto de aves pertenecen a otros órdenes y, en concreto, a las siguientes familias: Picidae (nueve especies); Accipitridae (tres especies); Strigidae, Falconidae, Cuculidae, Caprimulgidae, Apodidae y Coraciidae (dos especies cada una); y Tytonidae (una especie).

Uno de los aspectos un tanto sorprendentes de la RO consiste en la utilización, en más de una ocasión, de sinonimias ya en desuso entre buena parte de la comunidad científica de finales del siglo XIX. Así ocurre, entre varios ejemplos posibles, con el uso de los géneros Butaetes (para Buteo lagopus) o Apternus (para Picoides tridactylus), presentes de forma preferente en algunos tratados clásicos publicados en la primera mitad del siglo (Bonaparte, 1838), pero que aparecen relegados a un segundo plano en grandes obras posteriores (Degland \& Gerbe, 1867) y, en general, en la mayoría de las publicaciones de los ornitólogos españoles del siglo XIX (Reyes, 1886; Arévalo, 1887). En esta línea, Peña (1905) no dudó en variar por su cuenta la denominación científica de algunas especies de la RO, entre ellas el género de Buteo lagopus.

Pero más allá de cuestiones referentes a la nomenclatura científica utilizada en la RO, existen una serie de aspectos llamativos en cuanto a las especies contenidas en esta norma. Sin duda, entre los más evidentes se encuentra la presencia de algunas que no pertenecen a la avifauna española o que resultan infrecuentes, y que no pueden justificarse teniendo en cuenta el nivel de conocimientos de la época. Así, por ejemplo, la inclusión del ya mencionado Buteo lagopus en la RO constituye un innegable desacierto. Esta especie está considerada actualmente como accidental en la península ibérica (Díaz et al., 1996) $\mathrm{y}$, de hecho, ni siquiera aparecía en el Prontuario de la avifauna española (Bernis, 1954). Pero ya Arévalo (1887) señalaba que, aunque A. Machado, en 1854, y E. Vayreda, en 1883, citaran a esta rapaz como sedentaria y frecuente en varias 
localidades españolas, tal circunstancia debía ser una equivocación. Otro ejemplo interesante lo constituye la aparición del anteriormente comentado género Apternus, del que Arévalo (1887), con buen criterio, no citaba ningún representante en la avifauna española, aunque sí lo hacía Reyes (1886), al indicar la presencia de Picoides tridactylus en el noreste de España. Reyes, en realidad, no hacía más que repetir el error de Vayreda en su Fauna ornitológica de la provincia de Gerona, publicado en 1883, y en muchos aspectos carente de rigor (Salas et al., 2005).

Por contra, hay una serie de ausencias incomprensibles en familias constituidas por especies total o parcialmente insectívoras y, por tanto, «útiles a la agricultura», por lo que hubiera sido esperable encontrar incluidas en la RO a todos sus representantes con presencia conocida en la península ibérica, y no únicamente a algunos. Por ejemplo, se excluyeron a los siguientes taxones: Motacilla cinerea, Ptyonoprogne rupestris, Sylvia melanocephala y Sylvia undata. De todas estas aves, Reyes (1886) y Arévalo (1887) se hacían eco de su existencia en España, mediante datos propios o, también, de diferentes autores (Á. Guirao, F. Ríos Naceyro, H. Saunders, I. Vidal, L. H. L. Irby, V. López-Seoane, etc.). De Sylvia undata, en concreto, Arévalo refería la obtención años atrás de nidos de esta especie por diversos naturalistas locales, y de su comunicación al conocido ornitólogo británico Lord Lilford. Además, de Sylvia melanocephala se señalaba expresamente su presencia en España, incluso, en tratados más generales (Degland \& Gerbe, 1867).

No obstante, se podría argumentar que las ausencias de algunas otras especies en la RO quizá estarían justificadas por la dificultad de su identificación, y por su posición taxonómica en aquel tiempo aparentemente poco clara. Es el caso, por ejemplo, de Apus pallidus. Pero aun reconociendo que esta ave se había descubierto para la ciencia hacía relativamente pocos años (Shelley, 1871), lo cierto es que ya Arévalo (1887) la consideraba muy abundante en Andalucía, y la describía pormenorizadamente; y también Reyes (1886) comentaba su cita por $\mathrm{H}$. Saunders en 1872. Sin embargo, otras ausencias sí pueden resultar más comprensibles, como es el caso de Saxicola dacotiae, especie endémica de la isla canaria de Fuerteventura, pero que fue descrita por primera vez únicamente siete años antes de la publicación de la RO (Meade-Waldo, 1889).

En otro orden de cosas, en la propia RO se expone que la redacción y propuesta del catálogo de aves protegidas fue tarea del Consejo Superior de Agricultura, Industria y Comercio (en adelante CSA), organismo dependiente del Ministerio de Fomento y al que pocos naturalistas de prestigio pertenecieron, con una notable excepción: Mariano de la Paz Graells; este ilustre científico español formó parte de esta institución (Martín \& Izquierdo, 2009). Es conocido el interés de Graells por el fomento y la protección de los animales útiles, su esfuerzo por contribuir a catalogar la fauna y flora española, así como su dedicación al estudio de los más variados aspectos de las ciencias naturales, pudiéndosele considerar como uno de los últimos naturalistas totales (Izquierdo \& Martín, 2008; Casado, 2009; Cervantes, 2009). Por todo ello, no tiene nada de extraño que Graells participase activamente en la elaboración de la RO.

Los documentos que prueban esta vinculación y que aquí se muestran por primera vez se encuentran en el AMRM (signatura: 238/B; carpeta 1; años: 1892-1901), en las actas correspondientes al CSA. En ellas se pone de manifiesto que Graells fue uno de los dos vocales a los que, en la sesión del 4 de noviembre de 1896, se les encomendó la elaboración del listado de aves a proteger mediante su inclusión en una futura real orden ministerial. De hecho, el otro vocal -F. J. de Palacio y García de Velasco, conde de las Almenas- reconoció en la misma sesión la manifiesta y superior autoridad de Graells en esa materia, y todos convinieron en emplear como base para el listado de aves las que aparecían en la conferencia titulada Los aliados del labrador en su lucha entomológica, impartida por el propio Graells en el Jardín Botánico de Madrid, el 21 de junio de 1882. En la siguiente sesión del CSA - presidida por F. García Gómez de la Serna, el día 11 de noviembre de 1896-, se aprobó la relación de aves presentada por Graells, quien afirmó que se trataba de una «clasificación interina», cuyo objetivo era solventar rápidamente las dudas y problemas suscitados por la promulgación, unos meses antes, de la Ley de 19 de septiembre de 1896, de protección de los pájaros. Apenas un mes más tarde, el listado se publicó en la Gaceta de Madrid mediante una real orden ministerial (Ministerio de Fomento, 1896).

Dada la premura existente entonces, es disculpable que Graells cometiera los errores comentados anteriormente al redactar la RO, o que tal vez no valorase adecuadamente la memorable obra de José Arévalo - publicada con posterioridad a la mencionada conferencia en el Jardín Botánico de Madrid-, 
o la de otros destacables naturalistas. Asimismo, hay que tener en cuenta que el único trabajo importante de Graells en el ámbito de la ornitología lo había realizado bastantes decenios antes de que la RO viera la luz (Graells, 1853), y ya se revelaba entonces cierto capricho de autor frente a la habitual taxonomía empleada por sus contemporáneos (Bernis, 1998). Además, la mayoría de los investigadores actuales señalan que, aun siendo indiscutible la importancia del conjunto de su obra, sus últimos años de vida indican un cierto distanciamiento con respecto a las corrientes y avances científicos del momento (e.g., Aragón, 2009).

En cualquier caso, y a pesar de los defectos que puedan encontrarse en la $\mathrm{RO}$, es posible afirmar que otra de las grandes realizaciones de Graells fue la de conseguir que, en España, se aprobase por primera vez un catálogo de especies absolutamente amparadas por la ley y, con ello, logró situar a su país a la altura de las más importantes naciones europeas en materia de conservación de la fauna silvestre. Su fallecimiento en 1898 le impidió mejorarlo -como parece que era su deseo, según se puede inferir de las referidas actas del CSA del 4 y 11 de noviembre de 1896-, y la «clasificación interina» de aves protegidas perduró durante décadas, sin cambios, en el ordenamiento jurídico español.

\section{Agradecimientos}

Quiero expresar mi más sincero agradecimiento al Dr. Luis Miguel Torres Vila, por sus acertados comentarios al manuscrito inicial y por haberme mostrado lo apasionante que resulta la historia de las ciencias naturales. También quiero agradecer a M. ${ }^{a}$ del Carmen de San José Moreno y a Miguel Ángel de San José Lancha, por su inestimable e insustituible apoyo en todo momento; y a Marta García Serrano, por su indispensable ayuda en la traducción del Resumen al inglés.

\section{Referencias}

Aragón, S., 2009. La incorporación del evolucionismo en España. Quercus, 276: 24-29.

Arévalo, J., 1887. Aves de España (memorias de la Real Academia de Ciencias Exactas, Físicas y Naturales, tomo XI). Imprenta de viuda e hijo de Aguado. Madrid. 474 pp.

Barclay-Smith, P., 1959. The British contribution to bird protection. Ibis, 101(1): 115-122.

Bernis, F., 1954. Prontuario de la avifauna española (incluyendo aves de Portugal, Baleares y Canarias). Ardeola, 1: 11-85.
Bernis, F., 1998. De las Ciencias Naturales y la Zoología, antes, durante y poco después de fundarse la Sociedad Española de Historia Natural (con especial referencia a vertebrados terrestres). Memorias de la Real Sociedad Española de Historia Natural. 2. ${ }^{a}$ época, 1: 97-122.

Boardman, R., 2006. The international politics of bird conservation: biodiversity, regionalism and global governance. Edward Elgar Publishing. Cheltenham. $265 \mathrm{pp}$.

Bonaparte, C. L., 1838. A geographical and comparative list of the birds of Europe and North America. J. Van Voorst. London. 67 pp.

Calleja, T., 1926. Legislación de caza. Protección de los pájaros y uso de las armas. Góngora. Madrid. 325 pp.

Casado, S., 2009. Mariano de la Paz Graells, el último naturalista total. Quercus, 70: 30-33.

Cervantes, E., 2009 (coord.). El naturalista en su siglo: homenaje a Mariano de la Paz Graells en el CC aniversario de su nacimiento. Instituto de Estudios Riojanos. Logroño. 279 pp.

Degland, C. D. \& Gerbe, Z., 1867. Ornithologie européenne ou Catalogue descriptif, analytique et raisonné des oiseaux observés en Europe. Deuxième édition. Tome I. J. B. Baillière \& fils. Paris. 607 pp.

Díaz, M., Asensio, B. \& Tellería, J. L., 1996. Aves Ibéricas. I. No paseriformes. J. M. Reyero Editor. Madrid. 303 pp.

Ferrero-García, J. J., 2010. La conservación de las aves en la España de la Restauración. Quercus, 294: 2229.

Garrido, M., 2006. José Arévalo Baca (1844-90) y la ornitología en Málaga. En: Actas del IX Congreso de la Sociedad Española de Historia de las Ciencias y de las Técnicas. Tomo II. Sociedad Española de Historia de las Ciencias y de las Técnicas. Cádiz: 1033-1044.

Graells, M., 1853. Catálogo metódico de las aves observadas hasta el día en el área de la Fauna Matritense. En: Memorias de la Comisión del Mapa Geológico, Año de 1852. Madrid: 73-86.

Irby, L. H. L., 1895. The Ornithology of the Straits of

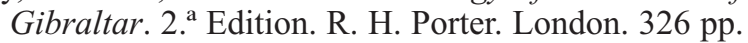

Izquierdo, I. \& Martín, C., 2008. Graells y Agenjo en GRAELLSIA. Graellsia, 64(2): 349-350.

Kettilby, G., 1869. The statutes of the United Kingdom of Great Britain and Ireland, 32 \& 33 Victoria, 1868-9. George E. Eyre \& William Spottiswoode. London. $752 \mathrm{pp}$.

MacPherson, A. H., 1909. Comparative legislation for the protection of birds. In: A. H. MacPherson \& G. A. Momber: Legislation for the protection of birds. The Royal Society for the Protection of Birds. London: 150. 
Marchant, J. R. V. \& Watkins, W., 2009. Wild birds Protection Acts, 1880-1896. BiblioLife. Charleston. 196 pp.

Martín, C. \& Izquierdo, I., 2009. Memorias, catálogos, prontuarios, manuales... la obra impresa de Mariano de la Paz Graells. En: E. Cervantes (coord.). El naturalista en su siglo: homenaje a Mariano de la Paz Graells en el CC aniversario de su nacimiento. Instituto de Estudios Riojanos. Logroño: 29-58.

Meade-Waldo, E. G., 1889. Further notes on the birds of de Canary Islands. Ibis, 31(4): 503-520.

Ministerio de Fomento, 1896. Real Orden de 25 de noviembre de 1896 , por la que se aprueba un catálogo de las aves cuya caza debe prohibirse en todo tiempo. Gaceta de Madrid, 337 (2 de diciembre de 1896): 831-832.

Oustalet, E., 1884. Rapport a M. le Ministre de l'instruction publique et des Beaux-arts sur le congrès et l'exposition ornithologiques de Vienne, en 1884. Burlin \& Cie. Angers. 406 pp.

Peña, A., 1905. Tratado de las aves insectívoras cuya caza está prohibida en España. Imprenta de Henrich y Com. ${ }^{a}$ en comandita. Barcelona. 297 pp.

Reyes, V., 1886. Catálogo de las aves de España, Portugal é Islas Baleares. Anales de la Sociedad Española de Historia Natural, 15(1): 5-109.

Salas, M. Á., Ferrer, X. \& Rovira, J. V., 2005. Distribución del Pico Mediano en la Península Ibérica. Revisión histórica crítica (siglos XIX y XX). Observatorio medioambiental, 8: 175-195.

SEO, 1956. El nuevo convenio internacional para la protección de las aves. Ardeola, 2(2): 241-248.

Shelley, G. E., 1871. Contributions to the Ornithology of Egypt. Ibis, 13(1): 38-54.

Tellería, J. L., 2004. Francisco Bernis y la ornitología española. En: La Ornitología hoy: homenaje al profesor Francisco Bernis Madrazo. Editorial Complutense. Madrid: 3-18.

Temminck, C. J., 1815. Manuel d'ornithologie ou tableau systématique des oiseaux qui se trouvent en Europe. J.C. Sepp \& G. Dufour. Amsterdam, Paris. $618 \mathrm{pp}$.

Torres-Vila, L. M., Ferrero-García, J. J., Martín, D. \& Sánchez, Á., 2009. La sanidad vegetal en Extremadura en el siglo XIX. En: Dioses, mitos y demonios: la agricultura extremeña en el siglo XIX. Colección: historia agraria y rural. Consejería de Agricultura y Desarrollo Rural, Junta de Extremadura. Badajoz: 97-114.

van Heijnsbergen, P., 1997. International legal protection of wild fauna and flora. IOS Press. Amsterdam. $280 \mathrm{pp}$.

\section{RESUMEN}

Se realiza un análisis crítico del número y composición de las aves estrictamente amparadas por la Real Orden de 25 de noviem bre de 1896, por la que se creaba, por primera vez en España, un catálogo de especies legalmente protegidas. Se trata de un listado elaborado por un organismo público, entre cuyos componentes se encontraba Mariano de la Paz Graells, un emblemático naturalista español del siglo XIX. Se ha investigado en fuentes bibliográficas y archivos, hasta encontrar las pruebas documentales que vinculan a este ilustre científico con la primera norma española de protección de la fauna silvestre.

Palabras clave: Protección de especies; aves; agricultura; siglo XIX; Graells.

\section{ABSTRACT}

The first Spanish catalogue of protected species (1896): analysis of its contents and Graells' autorship

A critical analysis of the number and composition of the birds that are strictly protected by the Royal Order of 25 November 1896 , in which a catalogue of legally protected species was created for the first time in Spain, has been performed. This is a list developed by a public body, one of whose members was Mariano de la Paz Graells, a remarkable $19^{\text {th }}$ century Spanish naturalist. The research has been carried out on bibliographic sources and archives, and its main result is the finding of the documentary evidence that proves the linkage of this illustrious scientist with the first Spanish regulation about wildlife protection.

Keywords: Species protection; birds; agriculture; $19^{\text {th }}$ century; Graells.

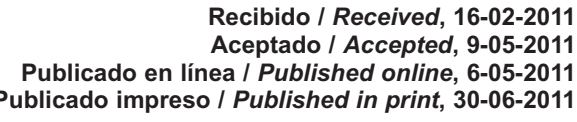

